SITZUNGSBERICHTE DER SÄCHSISCHEN AKADEMIE DER WISSENSCHAFTEN ZU LEIPZIG Mathematisch-naturwissenschaftliche Klasse Band $115 \cdot$ Heft 6

ERNST NEEF

DER VERLUST DER ANSCHAULICHKEIT IN DER GEOGRAPHIE UND DAS PROBLEM DER KULTURLANDSCHAFT

AKADEMIE-VERLAG • BERLIN 


\title{
SITZUNGSBERICHTE DER SÄCHSISCHEN AKADEMIE DER WISSENSCHAFTEN ZU LEIPZIG
}

\author{
MATHEMATISCH-NATURWISSENSCHAFTLICHE KLASSE
}

Band 109

Heft 1 Prof. Dr. ERICH RAMMLER, Uber die Theorien der Braunkohlenbrikettentstehung

$$
\text { 1970. } 38 \text { Seiten - } 13 \text { Abb., davon } 2 \text { auf } 2 \text { Tafeln }-8^{\circ}-\mathrm{M}_{4,-}
$$

Heft 2 Prof. Dr. WolfaANG TUTSCHKe, Stammfunktionen komplexwertiger Funktionen

$$
\text { 1970. } 20 \text { Seiten }-8^{\circ}-\text { M 3,70 }
$$

Heft 3 Dr. habil. GUNTHER EISENREICH, Zur Syzygientheorie und Theorie des inversen Systems perfekter Ideale und Vektormodule in Polynomringen und Stellenringen 1070.88 Seiten $-8^{\circ}-\mathrm{M} 10,80$

Heft 4 Prof. Dr. med. RolF FMMrich, Hochdruck und Hyperlipidämie (Hypercholesterinämie) als Risikofaktoren für die Entstehung der Arteriosklerose

$$
\text { 1971. } 23 \text { Seiten }-10 \text { Abbildungen }-4 \text { Tabellen }-8^{\circ}-\text { M } 3,90
$$

Heft 5 Prof. Dr. Hans DrisoheL, Biologische Rhythmen

$$
\text { 1972. 57 Seiten }-31 \text { Abbildungen }-1 \text { Tabelle }-8^{\circ}-\mathrm{M} 0,60
$$

Heft 6 Prof. Dr.-Ing. Dr. h. c. KURT SCHWaBE, Konzentrierte Elektrolytlösungen - Thermodynamische und kinetische Eigenschaften 1972. 49 Seiten -27 Abbildungen -2 Tabellen $-8^{\circ}-$ M 7,50

Heft 7 Prof. Dr. Wolfans TUTSснке, Konstruktion von globalen Lösungen mit vorgeschriebenen Singularitäten bei partiellen komplexen Differentialgleichungssystemen 1972. 24 Seiten $-8^{\circ}-\mathrm{M} \mathrm{4}_{4,50}$

Band 110

Heft 1 Prof. Dr. h. c. PaUL GörLIch, Uber die Laser und ihre Anwendung

1972. 24 Seiten $-8^{\circ}-$ M 2,30

Heft 2 Prof. Dr. Hasso EssBach, Zum Problem der Tumoren im Kindesalter 1972. 24 Seiten - 11 Abbildungen auf 10 Kunstdrucktafeln $-8^{\circ}-$ M 6,

Heft 3 Prof. Dr. med. WALTER BREDNow, Zur Anthropologie des Schwindels

$$
\text { 1973. 17 Seiten }-2 \text { Abbildungen auf } 2 \text { Kunstdrucktafeln }-8^{\circ}-\text { M 2,50 }
$$

Heft 4 Prof. Dr. h. c. PAUL GöRLICH, Betrachtungen über den Wissenschaftlichen Gerätebau

Heft 5 Prof. Dr. ERIOH RAMMLER, Einige Betrachtungen über Erdgas

1972. 39 Seiten $-8^{\circ}-$ M 3,-

$$
\text { 1974. } 43 \text { Seiten }-8 \text { Abbildungen }-3 \text { Tabellen }-8^{\circ}-\text { M 4,50 }
$$

Heft 6 Prof. Dr. Gustav E. R. Schulze, Zur Rolle des Einfachheitsprinzips im physikalischen Weltbild

$$
\text { 1974. } 23 \text { Seiten }-4 \text { Abbildungen - } 8^{\circ}-\text { M 2,50 }
$$

Heft 7 Prof. Dr. med. Rolf Emrrich, Zwischen Leben und Tod. Ärztliche Probleme der Thanatologie 1974. 22 Seiten -2 Abbildungen -4 Tabellen $-8^{\circ}-$ M 3,50

Band 111

Heft 1 Prof. Dr. Wilhelm MArER, Vom Erbe Bernhard Riemanns

1975. 16 Seiten $-8^{\circ}-$ M 2,50

Heft 2 Prof. Dr. med. HANS DRISCHEL, Organismus und geophysikalische Umwelt

1975. 50 Seiten -25 Abbildungen -1 Tabelle $-8^{\circ}-$ M 7,-

Heft 3 Prof. Dr. Maria HASSE, Zum Begriff des allgemeinen Produkts von Kategorien 